\title{
PARTITIONS, QUASIMODULAR FORMS, AND THE BLOCH-OKOUNKOV THEOREM
}

\author{
DON ZAGIER
}

In fond memory of Marvin Knopp, wonderful mathematician and friend, who made one feel happy to be human.

\begin{abstract}
We give a very short proof of the Bloch-Okounkov theorem on the quasimodularity of certain functions defined by sums over partitions, and also show how to make their map $\mathfrak{s l}_{2}$-equivariant.
\end{abstract}

Keywords: modular forms, partitions, representations of the symmetric group Mathematics Subject Classification: Primary 05A17, Secondary 11F11, 05E10

1. Introduction and main results. Let $\mathscr{P}$ denote the set of all partitions. If $f: \mathscr{P} \rightarrow \mathbb{Q}$ is an arbitrary function, we define the " $q$-average" or " $q$-bracket" of $f$ as the formal power series

$$
\langle f\rangle_{q}=\frac{\sum_{\lambda \in \mathscr{P}} f(\lambda) q^{|\lambda|}}{\sum_{\lambda \in \mathscr{P}} q^{|\lambda|}} \in \mathbb{Q}[[q]]
$$

where $|\lambda|$ denotes the integer of which $\lambda$ is a partition. If $f$ has at most polynomial growth in $|\lambda|$, which will be the case for all of the functions we consider, then the series in the numerator and denominator converge for all $q \in \mathbb{C}$ of absolute value less than 1 and we can consider $\langle f\rangle_{q}$ as a holomorphic function in the complex upper half-plane $\mathfrak{H}$ by setting $q=\mathbf{e}(\tau):=e^{2 \pi i \tau}$ for $\tau \in \mathfrak{H}$. In the language of statistical physics, one can think of $\langle f\rangle_{q}$ as the expectation value of an observable $f$ in a statistical system whose states are labelled by partitions and where the state $\lambda$ has energy $|\lambda|$ and is weighted by $q^{|\lambda|}$; then $2 \pi \tau / i$ would correspond to the reciprocal of the temperature multiplied by Boltzmann's constant, and the denominator in (1) would be the partition function of the ensemble.

A wonderful result of Bloch and Okounkov [3] states that for a large class of functions $f$ ("shifted symmetric polynomials"; see $\S 3$ for details) the function $\langle f\rangle_{q}$ is a quasimodular form on the full modular group. (See $\S 2$ for the definition and main properties of quasimodular forms.) That there is a connection with modular and quasimodular forms is not really surprising, since the denominator in (1) equals $q^{1 / 24} / \eta(\tau)$, where $\eta(\tau)=q^{1 / 24} \prod_{n=1}^{\infty}\left(1-q^{n}\right)$ is the Dedekind eta-function, so that (1) can be written in the equivalent form

$$
\frac{1}{\eta(\tau)}\langle f\rangle_{q}=\sum_{\lambda \in \mathscr{P}} f(\lambda) q^{|\lambda|-\frac{1}{24}}
$$

and it is well known that the 24th power and the logarithmic derivative of $\eta$ are modular of weight 12 and quasimodular of weight 2, respectively. Formula (2), together with the well-known fact that derivatives of quasimodular forms are quasimodular, already proves that $\langle f\rangle_{q}$ is quasimodular (of mixed weight) when $f(\lambda)$ is a polynomial in $|\lambda|$. A less trivial example was discovered by Dijkgraaf [4] in the context of "mirror symmetry in dimension one," i.e., the problem of counting (in a suitable sense) generically ramified coverings of a 2-torus having a given degree $n$ and genus $g \geq 2$. The solution of this problem, which is a special case of the general Hurwitz counting problem, is given in terms of the $q$-bracket $\left\langle\nu_{T}^{2 g-2}\right\rangle_{q}$, where $\nu_{T}$ is the function that associates to a partition $\lambda$ of $n$ the value (constant, by Schur's lemma) of the action of the sum of all transpositions in $\mathfrak{S}_{n}$ on the irreducible representation of $\mathfrak{S}_{n}$ corresponding to $\lambda$. An argument using the language and methods of mathematical physics (see [4] and 
Rudd [8]) indicated that this $q$-bracket should be a quasimodular form of weight $6 g-6$, and a proof of this fact in mathematical language was given by Kaneko and myself in [6].

The theorem of Bloch and Okounkov is a vast generalization of this special result. For each integer $k \geq 0$ one defines a certain function $Q_{k}: \mathscr{P} \rightarrow \mathbb{Q}$, the first four of these being

$$
Q_{0}(\lambda)=1, \quad Q_{1}(\lambda)=0, \quad Q_{2}(\lambda)=|\lambda|-\frac{1}{24}, \quad Q_{3}(\lambda)=\nu_{T}(\lambda)
$$

with $\nu_{T}$ as above. (The definition will be recalled in $\S 3$. Our notations differ from those of [3].) We use the same letter $Q_{k}$ to denote both this function and the corresponding generator of the formal polynomial algebra

$$
\mathscr{R}=\mathbb{Q}\left[Q_{1}, Q_{2}, \ldots\right]
$$

in infinitely many variables; then any element $f=f\left(Q_{1}, Q_{2}, \ldots\right) \in \mathscr{R}$ can be considered as a function on partitions by setting $f(\lambda)=f\left(Q_{1}(\lambda), Q_{2}(\lambda), \ldots\right)$ and we can speak of its $q$-bracket $\langle f\rangle_{q}$. The result of Bloch and Okounkov is that this $q$-bracket is always a quasimodular form on the full modular group, of the same weight as $f$ if $f$ is homogeneous, where $\mathscr{R}$ is graded by assigning to $Q_{k}$ the weight $k$. The special cases mentioned above correspond to taking $f$ in the subring of $\mathscr{R}$ generated by $Q_{2}$ and $Q_{3}$.

Bloch and Okounkov gave two proofs of their theorem, a computational one closely following the proof in [6] for the special case $f=Q_{3}^{m}$ and a more conceptual one leading to an explicit formula for all $q$-brackets in terms of determinants of matrices of derivatives of theta functions, but both were quite difficult. In this paper we will give a very short proof and some extensions of the theorem. Our main result is the following identity, which leads immediately to a recursive way of computing $\langle f\rangle_{q}$ as a quasimodular form for any shifted symmetric polynomial $f$.

Theorem 1. For any $f$ in the subring $\Lambda_{*}=\mathbb{Q}\left[Q_{2}, Q_{3}, \ldots\right]$ of $\mathscr{R}$ we have the identity

$$
\langle\theta(\boldsymbol{\partial}) f\rangle_{q}=0 \text {. }
$$

Here \langle\rangle$_{q}: \mathbb{Q}[[q]]^{\mathscr{P}} \rightarrow \mathbb{Q}[[q]]$ is defined by linearity, $\theta(z) \in \mathbb{Q}[[q]][[z]]$ is the Jacobi theta series

$$
\theta(z)=\sum_{\nu \in \mathbb{F}}(-1)^{[\nu]} e^{\nu z} q^{\nu^{2} / 2} \quad\left(\mathbb{F}:=\mathbb{Z}+\frac{1}{2}\right),
$$

and $\boldsymbol{\partial}: \mathscr{R} \rightarrow \mathscr{R}$ is the derivation defined on generators by $\boldsymbol{\partial}\left(Q_{k}\right)=Q_{k-1}$, with $Q_{0}=1$.

The proof, which will be given in $\S 4$ after two preliminary sections on quasimodular forms and on invariants on partitions, is extremely easy, because it turns out that when one uses the definitions (2) and (5) to write the left-hand side of (4) as a double sum over the indexing set $\mathbb{F} \times \mathscr{P}$, then the terms simply cancel in pairs under a simple (albeit not very obvious) involution on this set!

In $\S 6$ we will show how to deduce from Theorem 1 the explicit formula of Bloch and Okounkov for $q$-brackets mentioned above. The next two sections contain the proofs of the two following theorems giving properties of the $q$-bracket of an arbitrary element $f \in \mathscr{R}$ as a polynomial in the Eisenstein series $E_{2}$ with coefficients in the ring of modular forms: the first describes the leading term of this polynomial, and the second gives its derivative with respect to $E_{2}$.

Theorem 2. For any $k \geq 0$ and any $f \in \mathscr{R}_{2 k}$ we have

$$
\langle f\rangle_{q}=-\frac{(2 k-3) ! !}{(-12)^{k}} \mu(f) E_{2}^{k}+\left(\text { combination of lower powers of } E_{2}\right),
$$

where $\mu: \mathbb{R} \rightarrow \mathbb{Q}$ is the ring homomorphism defined by $\mu\left(Q_{n}\right)=(1-n) / n$ ! and where $(2 k-3) !$ ! denotes the "double factorial" $1 \times 3 \times \cdots \times(2 k-3)$ (with $(-1) ! !=1$ and $(-3) ! !=-1)$. 
Theorem 3. Let $\mathfrak{d}$ be the derivation on quasimodular forms sending modular forms to zero and $E_{2}$ to 12 . Then

$$
\mathfrak{d}\langle f\rangle_{q}=\left\langle\frac{1}{2}\left(\mathscr{D}-\partial^{2}\right) f\right\rangle_{q}
$$

for all $f \in \mathscr{R}$, where $\boldsymbol{\partial}$ is as in Theorem 1 and $\mathscr{D}: \mathscr{R} \rightarrow \mathscr{R}$ is the second order differential operator of degree -2 defined by

$$
\mathscr{D}=\sum_{k, \ell \geq 0}\left(\begin{array}{c}
k+\ell \\
k
\end{array}\right) Q_{k+\ell} \frac{\partial^{2}}{\partial Q_{k+1} \partial Q_{\ell+1}} .
$$

We use this result to define an $\mathfrak{s l}_{2}$-action on $\mathscr{R}$ in such a way as to make the $q$-bracket map equivariant (Theorem 6). Finally, in $\S 9$ we will show that, rather surprisingly, the space of functions on $\mathscr{P}$ whose $q$-brackets are quasimodular is much larger than the ring $\mathscr{R}$ and contains many other interesting elements, and also (again somewhat unexpectedly) that this larger space is not closed under multiplication.

2. Quasimodular forms. We denote by $M_{*}=\bigoplus M_{k}$ and $\widetilde{M}_{*}=\bigoplus \widetilde{M}_{k}$ the rings of modular and quasimodular forms, respectively, on the full modular group $\Gamma=\operatorname{SL}(2, \mathbb{Z})$ with rational Fourier coefficients. We recall that an element of $M_{k}$ is a holomorphic function $\phi$ on the complex upper half-plane $\mathfrak{H}$ satisfying $\left.\phi\right|_{k} \gamma=\phi$ for all $\gamma \in \Gamma$ and having a Fourier expansion $\phi(\tau)=\sum_{n=0}^{\infty} a_{n} q^{n}$ with coefficients $a_{n} \in \mathbb{Q}$ of polynomial growth, where $\left.\phi\right|_{k} \gamma: \mathfrak{H} \rightarrow \mathbb{C}$ for $\gamma=\left(\begin{array}{ll}a & b \\ c & d\end{array}\right) \in \Gamma$ is the function sending $\tau \in \mathfrak{H}$ to $(c \tau+d)^{-k} \phi\left(\frac{a \tau+b}{c \tau+d}\right)$ and where $q=\mathbf{e}(\tau):=e^{2 \pi i \tau}$, while a function $\phi \in \widetilde{M}_{k}$ is a holomorphic function given by a Fourier expansion with the same growth condition but satisfying the weaker transformation property that $\left.\gamma \mapsto \phi\right|_{k} \gamma(\tau)$ is a polynomial in $\frac{c}{c \tau+d}$ for $\tau \in \mathfrak{H}$ fixed and $\gamma=\left(\begin{array}{ll}a & b \\ c & d\end{array}\right) \in \Gamma$ variable. (This is not the definition given in [6], but an equivalent one suggested by Werner Nahm. For more details, see $\S 5.3$ of [9].) The simplest example is the Eisenstein series

$$
G_{k}(\tau)=-\frac{B_{k}}{2 k}+\sum_{n=1}^{\infty} n^{k-1} \frac{q^{n}}{1-q^{n}} \quad\left(k>0 \text { even, } B_{k}=k \text { th Bernoulli number }\right)
$$

which is a modular form of weight $k$ if $k>2$ and a quasimodular form of weight 2 if $k=2$. Both $M_{*}$ and $\widetilde{M}_{*}$ are rings and it is well-known that

$$
M_{*}=\mathbb{Q}[Q, R], \quad \widetilde{M}_{*}=M_{*}[P]=\mathbb{Q}[P, Q, R],
$$

where $P, Q$ and $R$ (Ramanujan's notations for $E_{2}, E_{4}, E_{6}$ ) are the normalized Eisenstein series

$$
P=-24 G_{2}=1-24 q-\cdots, \quad Q=240 G_{4}=1+240 q+\cdots, \quad R=-504 G_{6}=1-504 q-\cdots .
$$

For example, we have $G_{8}=\frac{Q^{2}}{480}, G_{10}=-\frac{Q R}{264}$ and $G_{12}=\frac{441 Q^{3}+250 R^{2}}{65520}$.

A basic fact is that the ring $\widetilde{M}_{*}$ is closed under the differentiation operator

$$
D=\frac{1}{2 \pi i} \frac{d}{d \tau}=q \frac{d}{d q},
$$

as can be seen either from the definition or from (10) together with Ramanujan's formulas

$$
D(P)=\frac{P^{2}-Q}{12}, \quad D(Q)=\frac{P Q-R}{3}, \quad D(R)=\frac{P R-Q^{2}}{2} .
$$


The operator $D$ acts on $\widetilde{M}_{*}$ as a derivation of degree +2 (i.e., it raises the weight of a quasimodular form by 2). There are two further derivations $W$ and $\mathfrak{d}$ on $\widetilde{M}_{*}$ of degree 0 and -2 , respectively, defined by $W(\phi)=k \phi$ for $\phi \in \widetilde{M}_{k}$ (weight operator or Hamiltonian) and by $\mathfrak{d}(\phi)=12 F^{\prime}(P)$ if $\phi \in \widetilde{M}_{*}$ has been written using (10) as $F(P)$ where $F$ is a polynomial with coefficients in $M_{*}$. These three operators satisfy the commutation relations

$$
[W, D]=2 D, \quad[W, \mathfrak{d}]=-2 \mathfrak{d}, \quad[\mathfrak{d}, D]=W
$$

i.e., they span a 3-dimensional Lie algebra of derivations isomorphic to $\mathfrak{s l}_{2}$.

As well as the Eisenstein series, we will need another sequence of quasimodular forms $\left\{H_{n}\right\}_{n \geq 0}$, where $H_{n}$ has weight $n$ (and hence is zero if $n$ is odd). They can be defined inductively by

$$
H_{0}=1, \quad H_{1}=0, \quad 4 n(n+1) H_{n}=8 D\left(H_{n-2}\right)+P H_{n-2} \quad \text { if } n>1
$$

(or in closed form $\left.H_{2 r}=\frac{1}{(2 r+1) !}\left(2 D+\frac{1}{4} P\right)^{r}(1)\right)$, the first few non-zero values being

$$
H_{0}=1, \quad H_{2}=\frac{P}{24}, \quad H_{4}=\frac{5 P^{2}-2 Q}{5760}, \quad H_{6}=\frac{35 P^{3}-42 P Q+16 R}{2903040} .
$$

A more natural definition of these forms is by the Taylor expansion

$$
\frac{\theta(z)}{\theta^{\prime}(0)}=\sum_{n=0}^{\infty} H_{n}(\tau) z^{n+1},
$$

where $\theta(z)=\theta(z ; \tau)$ is the Jacobi theta series defined by (5). To see the equivalence of these two definitions, note that if we define $H_{n}$ by (14) then from (5) we get

$$
(2 r+1) ! \theta^{\prime}(0) H_{2 r}=\sum_{\nu \in \mathbb{F}}(-1)^{[\nu]} \nu^{2 r+1} q^{\nu^{2} / 2}=(2 D)^{r} \theta^{\prime}(0)
$$

and (13) then follows from Jacobi's formula $\theta^{\prime}(0)=\eta^{3}$ (here $\eta(\tau)$ is the Dedekind eta-function as in $\S 1$, with $\left.\eta^{24}=\left(Q^{3}-R^{2}\right) / 1728\right)$ together with the differentiation formula $D(\eta)=\frac{1}{24} P \eta$. Yet a third way to compute the functions $H_{n}$ is by using the formula

$$
z \frac{\theta^{\prime}(z)}{\theta(z)}=1-2 \sum_{k>0} G_{k} \frac{z^{k}}{k !}
$$

(which follows from the Jacobi triple product) to get the identity $n H_{n}=-2 \sum_{0<k \leq n} G_{k} H_{n-k} / k$ ! for all $n$, which together with the same initial conditions as in (13) determines all of the $H_{n}$ recursively in terms of the Eisenstein series $G_{k}$. From formula (16) and the modularity of $G_{k}$ for $k>2$ we see easily that $\sum H_{n} z^{n}=e^{P z^{2} / 24} \sum h_{n} z^{n}$ or $H_{n}=\sum_{r=0}^{n} h_{n-2 r}(P / 24)^{r} / r$ ! where the $h_{n}\left(h_{0}=1\right.$, $\left.h_{2}=0, h_{4}=-Q / 2880, h_{6}=R / 181440, \ldots\right)$ are now modular rather than quasimodular forms, given recursively by $\left(\begin{array}{c}n+3 \\ 2\end{array}\right) h_{n+2}=\left(D-\frac{n}{12} P\right) h_{n}-\frac{1}{288} Q h_{n-2}$.

3. Partitions and shifted symmetric polynomials. As in the introduction, we denote by $\mathscr{P}$ the set of all partitions. Each partition is represented as $\lambda=\left(\lambda_{1}, \lambda_{2}, \ldots\right)$ with $\lambda_{1} \geq \lambda_{2} \geq \cdots$ and $\lambda_{j}=0$ for all but finitely many $j$, or equivalently by a Young diagram $Y_{\lambda}$ whose $j$ th row consists of $\lambda_{j}$ boxes, 
with total size $|\lambda|=\sum_{j \geq 1} \lambda_{j}$. Following Bloch and Okounkov (though with different notations), for all $\lambda \in \mathscr{P}$ we will now define invariants $P_{k}(\lambda) \in \mathbb{Z}\left[\frac{1}{2}\right]$ and $Q_{k}(\lambda) \in \mathbb{Q}(k \geq 0)$, related by

$$
Q_{0}(\lambda)=1, \quad Q_{k}(\lambda)=\frac{P_{k-1}(\lambda)}{(k-1) !}+\beta_{k} \quad \text { if } k \geq 1
$$

where $\beta_{0}=1, \beta_{1}=0, \beta_{2}=-\frac{1}{24}, \ldots$ are defined by the power series expansion

$$
\frac{z / 2}{\sinh (z / 2)}=\sum_{n=0}^{\infty} \beta_{n} z^{n}
$$

The numbers $P_{k}(\lambda)$ are most conveniently defined in terms of the Frobenius coordinates of $\lambda$. These are the numbers $\left(r ; a_{1}, \ldots, a_{r} ; b_{1}, \ldots, b_{r}\right)$ where $r$ is the length of the longest principal diagonal contained in the Young diagram of $\lambda$ (or, in more modern bibliometric terms, the $h$-index of a researcher whose $j$ th most cited paper has $\lambda_{j}$ citations) and the numbers $a_{1}>\cdots>a_{r} \geq 0$ and $b_{1}>\cdots>b_{r} \geq 0$ are the arm- and leg-lengths (=number of cells to the right of, resp. below) of the cells on this diagonal (see

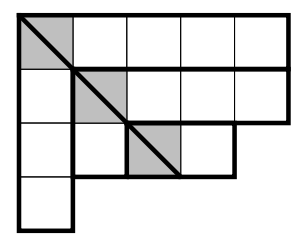

Partition $\lambda=(5,5,4,1)$

Frobenius coordinates $(3 ; 4,3,1 ; 3,1,0)$

$C_{\lambda}=\left\{-\frac{7}{2},-\frac{3}{2},-\frac{1}{2}, \frac{3}{2}, \frac{7}{2}, \frac{9}{2}\right\}$

$\left(P_{0}, P_{1}, P_{2}, \ldots\right)=\left(0,15,20, \frac{735}{4}, 410, \frac{46575}{16}, \ldots\right)$

diagram). This gives us the "balanced fermionic set"

$$
C_{\lambda}:=\left\{-b_{1}-\frac{1}{2}, \ldots,-b_{r}-\frac{1}{2}, a_{r}+\frac{1}{2}, \ldots, a_{1}+\frac{1}{2}\right\} \subset \mathbb{F},
$$

where $\mathbb{F}=\mathbb{Z}+\frac{1}{2}$ ("fermions") as in (5). We then define the invariants $P_{k}(\lambda)(k \geq 0)$ by

$$
P_{k}(\lambda)=\sum_{c \in C_{\lambda}} \operatorname{sgn}(c) c^{k}=\sum_{i=1}^{r}\left[\left(a_{i}+\frac{1}{2}\right)^{k}-\left(-b_{i}-\frac{1}{2}\right)^{k}\right],
$$

the first two cases being

$$
P_{0}(\lambda)=\sum_{c \in C_{\lambda}} \operatorname{sgn}(c)=0, \quad P_{1}(\lambda)=\sum_{c \in C_{\lambda}}|c|=\sum_{i=1}^{r}\left(a_{i}+b_{i}+1\right)=|\lambda| .
$$

To define the invariants $Q_{k}(\lambda)$ we associate to $\lambda \in \mathscr{P}$ the set $X_{\lambda}=\left\{\lambda_{j}-j+\frac{1}{2} \mid j \geq 1\right\} \subset \mathbb{F}$. It is easily checked that the sets $X_{\lambda}$ and $C_{\lambda}$ are related by

$$
\mathbb{F}^{+} \cap X_{\lambda}=C_{\lambda}^{+}=\left\{a_{r}+\frac{1}{2}, \ldots, a_{1}+\frac{1}{2}\right\}, \quad \mathbb{F}^{-} \backslash X_{\lambda}=C_{\lambda}^{-}=\left\{-b_{1}-\frac{1}{2}, \ldots,-b_{r}-\frac{1}{2}\right\}
$$

and that the map $\lambda \mapsto X_{\lambda}$ gives a bijection between $\mathscr{P}$ and $X_{\mathbb{F}}^{0}$, where $X_{\mathbb{F}}$ denotes the set of all subsets $X \subset \mathbb{F}$ that are bounded above and whose complements are bounded below, and $X_{\mathbb{F}}^{0} \subset X_{\mathbb{F}}$ the subset of those $X$ for which the finite sets $\mathbb{F}^{+} \cap X$ and $\mathbb{F}^{-} \backslash X$ have the same cardinality. To any set $X \in X_{\mathbb{F}}$ we associate the formal generating series

$$
\left.w_{X}(T)=\sum_{x \in X} T^{x} \in T^{1 / 2} \mathbb{Z}\left[T, T^{-1}\right]\right] .
$$


This series clearly converges for $T>1$ and belongs to $\frac{T^{1 / 2}}{T-1}+T^{1 / 2} \mathbb{Z}\left[T^{-1}, T\right]$, so that it defines a meromorphic function $W_{X}(z)$ and a sequence of coefficients $\left\{Q_{k}(X)\right\}_{k \geq 0}$ by

$$
W_{X}(z):=w_{X}\left(e^{z}\right) \quad(z \in \mathbb{C} \backslash 2 \pi i \mathbb{Z}), \quad W_{X}(z)=: \sum_{k=0}^{\infty} Q_{k}(X) z^{k-1} \quad(0<|z|<2 \pi) .
$$

Thus $Q_{0}(X)$ is always equal to 1 and $Q_{1}(X)=\left|\mathbb{F}^{+} \cap X\right|-\left|\mathbb{F}^{-} \backslash X\right|$ is an integer that vanishes if and only if $X \in X_{\mathbb{F}}^{0}$. For $\lambda \in \mathscr{P}$ we define $Q_{k}(\lambda)=Q_{k}\left(X_{\lambda}\right)$ and $W_{\lambda}(z)=W_{X_{\lambda}}(z)=\sum_{k>0} Q_{k}(\lambda) z^{k-1}$, and observe that the relation (21) immediately implies the relation (17), since $\frac{T^{1 / 2}}{T-1}=\frac{z / 2}{\sinh (z / 2)}$ for $T=e^{z}$. In the example $\lambda=(5,5,4,1,0,0, \ldots)$ illustrated above, we find:

$$
\begin{aligned}
X_{\lambda} & =\left\{\frac{9}{2}, \frac{7}{2}, \frac{3}{2},-\frac{5}{2},-\frac{9}{2},-\frac{11}{2}, \ldots\right\} \\
W_{\lambda}(z) & =e^{9 z / 2}+e^{7 z / 2}+e^{3 z / 2}+e^{-5 z / 2}+e^{-9 z / 2}\left(1-e^{-z}\right)^{-1}, \\
& =z^{-1}+\frac{359}{24} z+10 z^{2}+\frac{176407}{5760} z^{3}+\frac{205}{12} z^{4}+\frac{23473769}{967680} z^{5}+\cdots \\
& =\frac{1 / 2}{\sinh z / 2}+15 z+10 z^{2}+\frac{245}{8} z^{3}+\frac{205}{12} z^{4}+\frac{3105}{128} z^{5}+\cdots,
\end{aligned}
$$

and we recover the values of $P_{k}(\lambda)$ given before.

To any element $f \in \mathbb{R}=\mathbb{Q}\left[Q_{1}, Q_{2}, \ldots\right]$ we associate functions (which we denote by the same letter) $f: X_{\mathbb{F}} \rightarrow \mathbb{Q}$ and $f: \mathscr{P} \rightarrow \mathbb{Q}$ by

$$
f(X)=f\left(Q_{1}(X), Q_{2}(X), \ldots\right), \quad f(\lambda)=f\left(X_{\lambda}\right)=f\left(Q_{1}(\lambda), Q_{2}(\lambda), \ldots\right)
$$

(these functions are called shifted symmetric polynomials) and hence also a $q$-bracket $\langle f\rangle_{q}$ as in (1). We have a similarly defined map $f: X_{\mathbb{Z}} \rightarrow \mathbb{Q}$, where $X_{\mathbb{Z}}$ is defined exactly like $X_{\mathbb{F}}$ but with $\mathbb{F}=\mathbb{Z}+\frac{1}{2}$ replaced by $\mathbb{Z}$ (so elements of $X_{\mathbb{Z}}$ are subsets of $\mathbb{Z}$ that are bounded above and cobounded below). The following simple result will be the key to our proof of the Bloch-Okounkov theorem.

Proposition 1. The map $(\nu, \lambda) \mapsto X=X_{\lambda}+\nu$ defines a bijection $\mathbb{F} \times \mathscr{P} \approx X_{\mathbb{Z}}$, with the invariants of $X$ and $\lambda$ related by $Q_{1}(X)=\nu, Q_{2}(X)=|\lambda|-\frac{1}{24}+\frac{\nu^{2}}{2}$, and more generally

$$
f(X)=\left(e^{\nu \boldsymbol{\partial}} f\right)(\lambda) \quad \text { for all } f \in \mathcal{R},
$$

where $\boldsymbol{\partial}: \mathscr{R} \rightarrow \mathscr{R}$ is the derivation of degree -1 defined in Theorem 1 .

Proof. Translating an element $X \in X_{\mathbb{F}} \cup X_{\mathbb{Z}}$ by $\nu \in \mathbb{F}$ clearly multiplies $w_{X}(T)$ by $T^{\nu}$, so the invariants of $X$ and $X+\nu$ are related by

$$
\sum_{k=0}^{\infty} Q_{k}(X+\nu) z^{k-1}=w_{X+\nu}\left(e^{z}\right)=e^{\nu z} w_{X}\left(e^{z}\right)=\sum_{k=0}^{\infty}\left(\sum_{j=0}^{k} Q_{k-j}(X) \frac{\nu^{j}}{j !}\right) z^{k-1}
$$

or $Q_{k}(X+\nu)=\left(e^{\nu \boldsymbol{\partial}} Q_{k}\right)(X)$. In particular $Q_{1}(X+\nu)=Q_{1}(X)+\nu$, so the map $(\nu, X) \mapsto X+\nu$ gives a bijection $\mathbb{F} \times X_{\mathbb{F}}^{0} \approx X_{\mathbb{Z}}$ (and hence also $\mathbb{F} \times \mathscr{P} \approx X_{\mathbb{Z}}$ ), with inverse $X \mapsto\left(Q_{1}(X), X-Q_{1}(X)\right.$ ). This proves the first statement of the proposition and also the second for $f=Q_{k}$, and this is enough since $f \mapsto e^{\nu \boldsymbol{\partial}} f$ and $f \mapsto f(X)$ are ring homomorphisms and the $Q_{k}$ generate the ring $\mathscr{R}$. 
4. A recursive formula for the Bloch-Okounkov bracket. In this section we prove Theorem 1 and show how it implies the Bloch-Okounkov theorem.

Proof of Theorem 1. From equations (2) and (5) and Proposition 1 we have

$$
\frac{\langle\theta(\boldsymbol{\partial}) f\rangle_{q}}{\eta(\tau)}=\sum_{\substack{\nu \in \mathbb{F} \\ \lambda \in \mathscr{P}}}(-1)^{[\nu]}\left(e^{\nu \boldsymbol{\partial}} f\right)(\lambda) q^{|\lambda|-1 / 24+\nu^{2} / 2}=\sum_{X \in \mathcal{X}_{\mathbb{Z}}}(-1)^{\left[Q_{1}(X)\right]} f(X) q^{Q_{2}(X)}
$$

for any $f \in \mathscr{R}$, and this vanishes if $f \in \Lambda_{*}$ because the involution $X_{\mathbb{Z}} \rightarrow X_{\mathbb{Z}}$ defined by

$$
X \mapsto X^{*}= \begin{cases}X \backslash\{0\} & \text { if } 0 \in X, \\ X \cup\{0\} & \text { if } 0 \notin X,\end{cases}
$$

changes $w_{X}(T)$ by \pm 1 and hence changes $Q_{1}(X)$ by \pm 1 and leaves $Q_{k}(X)$ unchanged for all $k \geq 2$, so that the terms in the final sum cancel in pairs.

Corollary (Bloch-Okounkov theorem). The q-bracket $\langle f\rangle_{q}$ belongs to $\widetilde{M}_{k}$ for any $f \in \mathscr{R}_{k}$.

Proof. The assertion is obvious for $k=0$ (because $\mathscr{R}_{0}=\mathbb{Q} \cdot 1$ and $\langle 1\rangle_{q}=1$ ) and for $f \in Q_{1} \mathscr{R}$ (because $Q_{1}(\lambda)=0$ for every partition), and one shows easily using a lexicographic ordering of the monomial basis that $\mathscr{R}_{k}$ for $k>0$ is the sum of $\boldsymbol{\partial}\left(\Lambda_{k+1}\right)$ and $Q_{1} \mathscr{R}_{k-1}$. Hence we can assume that $f=\boldsymbol{\partial}\left(f_{1}\right)$ for some $f_{1} \in \Lambda_{k+1}$. Then applying (4) to $f_{1}$ and using (14) gives the identity

$$
0=\sum_{n=0}^{\infty} H_{n}(\tau)\left\langle\boldsymbol{\partial}^{n}(f)\right\rangle_{q}=\langle f\rangle_{q}+\frac{P}{24}\left\langle\boldsymbol{\partial}^{2}(f)\right\rangle_{q}+\frac{5 P^{2}-2 Q}{5760}\left\langle\boldsymbol{\partial}^{4}(f)\right\rangle_{q}+\cdots
$$

This proves the statement by induction on $k$ since $\partial^{n}(f)$ has weight $k-n<k$ for $n>0$.

5. Examples and discussion. Before continuing, we digress to make a few remarks concerning various aspects of Theorem 1 and its proof.

The first comment is that the involution (25) used in the proof of the theorem corresponds under the bijection $X_{\mathbb{Z}} \approx \mathbb{F} \times \mathscr{P}$ to the bijection on $\mathbb{F} \times \mathscr{P}$ sending $(\nu, \lambda)$ to $\left(\nu^{*}, \lambda^{*}\right)$, where

$$
\left\{\begin{array}{lll}
\nu^{*}=\nu+1, & \lambda^{*}=\left(\lambda_{1}+1, \ldots, \lambda_{k-1}+1, \lambda_{k+1}, \lambda_{k+2}, \ldots\right) & \text { if }-\nu \in X_{\lambda}, \\
\nu^{*}=\nu-1, & \lambda^{*}=\left(\lambda_{1}-1, \ldots, \lambda_{k-1}-1, k+\nu-\frac{3}{2}, \lambda_{k}, \lambda_{k+1}, \ldots\right) & \text { if }-\nu \notin X_{\lambda},
\end{array}\right.
$$

with $k \geq 1$ defined by $\lambda_{k}-k+\frac{1}{2}=-\nu$ in the first case and by $\lambda_{k-1}-(k-1)+\frac{1}{2}>-\nu>\lambda_{k}-k+\frac{1}{2}$ (or simply $-\nu>\lambda_{1}-\frac{1}{2}$ if $k=1$ ) in the second. We originally found the above proof in terms of the complicated map $(\nu, \lambda) \mapsto\left(\nu^{*}, \lambda^{*}\right)$, which was discovered experimentally, but verifying that it is an involution and that it leaves the quantities $|\lambda|-\nu^{2} / 2$ and more generally $\left(e^{\nu \boldsymbol{\partial}} Q_{k}\right)(\lambda)(k \geq 2)$ invariant requires some work, and the version given here using the bijection of Proposition 1 is much simpler and more natural.

Secondly, we want to emphasize that the method of proof given here is completely algorithmic: any shifted symmetric polynomial of positive weight can be written explicitly, though non-uniquely, as the sum of a multiple of $Q_{1}$, whose $q$-bracket is 0 , and an element $f \in \partial\left(\Lambda_{*}\right)$, for which one can compute the $q$-bracket as $-\sum_{n>0} H_{n}\left\langle\boldsymbol{\partial}^{n} f\right\rangle_{q}$ by Theorem 1 . This is very effective and easy to program. As an illustration, we have included a small table of $q$-brackets in an appendix at the end of the paper. 
As an example of how such calculations work, we say a few words about the case of the $q$-brackets of powers of $Q_{3}=\nu_{T}$, i.e., the original Dijkgraaf example. Here Theorem 2 says that the expansion of the quasimodular form $A_{n}:=\left\langle Q_{3}^{2 n}\right\rangle_{q} \in \widetilde{M}_{6 n}$ begins

$$
A_{n}=(-1)^{n-1} \frac{(6 n-3) ! !}{2^{6 n} 3^{5 n}} E_{2}^{3 n}+\cdots,
$$

which was Theorem 2 of [6], but in fact we can use Theorem 1 to get a complete description of the functions $A_{n}$ via a recursive set of differential equations. To do this, we use that the subring $\mathbb{Q}\left[Q_{2}, Q_{3}\right]$ of $\mathscr{R}$ is closed under the derivation $\boldsymbol{\partial}$ and that multiplying an element of $\mathscr{R}$ by $Q_{2}$ corresponds to applying $D+G_{2}$ to its $q$-bracket (eq. (37) below), getting after a short calculation

$$
\sum_{m=0}^{n} L_{n-m}\left(A_{m} /(2 m) !\right)=0 \quad(n \geq 0)
$$

where $L_{r}: \widetilde{M}_{*} \rightarrow \widetilde{M}_{*+6 r+2}$ is the differential operator

$$
L_{r}=\sum_{s=0}^{2 r+1} \frac{(6 r-2 s+3) !}{6^{2 r-s+1}(2 r-s+1) ! s !} H_{6 r-2 s+2}\left(D+G_{2}\right)^{s} .
$$

Finally, we make a few remarks about the formalism for partitions explained in $\S 3$. The set $X_{\mathbb{F}}$ of subsets $X$ of $\mathbb{F}$ that are bounded above and whose complements are bounded below is reminiscent of Dirac's famous "sea" of negative energy particles, in which the vacuum corresponds to all negative energy states being occupied and all positive energy ones empty, and electrons and positrons correspond respectively to filled positive energy states and to "holes" in the sea of negative energy states. This model works because the electrons are fermionic and obey the Pauli exclusion principle, so the mathematical version of it is a vector space generated under creation and annihilation operators from a vacuum state $\cdots \wedge e_{-5 / 2} \wedge e_{-3 / 2} \wedge e_{-1 / 2}$ and in which each element $X \in X_{\mathbb{F}}$ corresponds to the infinite wedge $\bigwedge_{x \in X} e_{x}$. In fact, this corresponds to the point of view taken by Bloch and Okounkov, whose paper contains the words "infinite wedge representation" in its title and uses several notions coming from conformal field theory and other parts of mathematical physics. The definitions and arguments given in Sections 3 and 4 here are elementary and do not need any of these concepts, but they seemed worth mentioning. In the same vein, the interaction between subsets of $\mathbb{F}$ and $\mathbb{Z}$ that played a key role in the proof above may perhaps be viewed as a kind of very naive form of supersymmetry.

6. Comparison with the Bloch-Okounkov formula for $q$-brackets. Since the $Q_{k}$ generate the ring $\mathcal{R}$, knowing $\langle f\rangle_{q}$ for all $f \in \mathscr{R}$ is equivalent to knowing the generating function

$$
F_{n}\left(z_{1}, \ldots, z_{n}\right):=\left\langle W\left(z_{1}\right) \cdots W\left(z_{n}\right)\right\rangle_{q}=\sum_{k_{1}, \ldots, k_{n} \geq 0}\left\langle Q_{k_{1}} \cdots Q_{k_{n}}\right\rangle_{q} z_{1}^{k_{1}-1} \ldots z_{n}^{k_{n}-1}
$$

(a Laurent series in $n$ variables) for all $n \geq 0$. Here " $W\left(z_{i}\right)$ " is the function $\lambda \mapsto W_{\lambda}\left(z_{i}\right)$ from partitions to Laurent series in $z_{i}$, where $W_{\lambda}(z)=\sum_{k=0}^{\infty} Q_{k}(\lambda) z^{k-1}$ as in $\S 3$. Bloch and Okounkov give the value of the generating function (27) as the full symmetrization $\sum_{\pi \in \mathfrak{S}_{n}} V_{n}\left(z_{\pi(1)}, \cdots, z_{\pi(n)}\right)$ of a simpler function $V_{n}\left(z_{1}, \ldots, z_{n}\right)$ that is defined as the quotient of a certain determinant of derivatives of $\theta(z)$, evaluated at subsums $z=z_{1}+\cdots+z_{m}(0 \leq m \leq n)$, by the product $\prod_{m=1}^{n} \theta\left(z_{1}+\cdots+z_{m}\right)$. Essentially by applying Cramer's rule, they rewrite this formula for $V_{n}$ as the recursion

$$
\sum_{m=0}^{n} \frac{(-1)^{n-m}}{(n-m) !} \theta^{(n-m)}\left(z_{1}+\cdots+z_{m}\right) V_{m}\left(z_{1}, \ldots, z_{m}\right)=0 \quad \text { for all } n \geq 1
$$


(p. 30 of [3], with a sign corrected) with the initial condition $V_{0}()=1$, the first three cases being

$$
\begin{aligned}
V_{1}(z)=\frac{\theta^{\prime}(0)}{\theta(z)}, & V_{2}\left(z_{1}, z_{2}\right)=\frac{\theta^{\prime}\left(z_{1}\right) V_{1}\left(z_{1}\right)}{\theta\left(z_{1}+z_{2}\right)}=\frac{\theta^{\prime}(0)}{\theta\left(z_{1}+z_{2}\right)} \frac{\theta^{\prime}\left(z_{1}\right)}{\theta\left(z_{1}\right)} \\
V_{3}\left(z_{1}, z_{2}, z_{3}\right) & =\frac{\theta^{\prime}\left(z_{1}+z_{2}\right) V_{2}\left(z_{1}, z_{2}\right)-\frac{1}{2} \theta^{\prime \prime}\left(z_{1}\right) V_{1}\left(z_{1}\right)+\frac{1}{6} \theta^{\prime \prime \prime}(0)}{\theta\left(z_{1}+z_{2}+z_{3}\right)} \\
& =\frac{\theta^{\prime}(0)}{\theta\left(z_{1}+z_{2}+z_{3}\right)}\left(\frac{\theta^{\prime}\left(z_{1}\right)}{\theta\left(z_{1}\right)} \frac{\theta^{\prime}\left(z_{1}+z_{2}\right)}{\theta\left(z_{1}+z_{2}\right)}-\frac{1}{2} \frac{\theta^{\prime \prime}\left(z_{1}\right)}{\theta\left(z_{1}\right)}+\frac{E_{2}}{24}\right) .
\end{aligned}
$$

Symmetrizing the recursion (28) with respect to the $n$ variables $z_{1}, \ldots, z_{n}$, and observing that there are $(n-|I|)$ ! numberings of the elements of the complement of a subset $I$ of $\{1, \ldots, n\}$, we obtain the following equivalent formulation of Bloch and Okounkov's formula for the generating function $F_{n}$.

Theorem 4. The functions $F_{n}\left(z_{1}, \ldots, z_{n}\right)$ are determined recursively by the equations

$$
\sum_{I \subseteq\{1, \ldots, n\}}(-1)^{n-|I|} \theta^{(n-|I|)}\left(\sum_{i \in I} z_{i}\right) F_{|I|}\left(\left\{z_{i}\right\}_{i \in I}\right)=0 \quad(n \geq 1)
$$

together with the initial condition $F_{0}()=1$.

We will show in a moment that the recursion (31) is equivalent to Theorem 1 (which was in fact discovered in this way, with the easy direct proof noticed only afterwards). But we show first that we can also rewrite this recursion in the form of the following simple recursive axiomatic definition of the Laurent series $F_{n}$, where in the final axiom we have used the notation $[G]^{+}$to denote the strictlypositive-exponent part of a Laurent series $G$ in several variables.

Theorem 5. The functions $F_{n}\left(z_{1}, \ldots, z_{n}\right)(n \geq 0)$ are the unique Laurent series satisfying:

(i) $F_{0}()=1$.

(ii) $F_{n}\left(z_{1}, \ldots, z_{n}\right)$ is symmetric in all $n$ arguments.

(iii) $F_{n}\left(z_{1}, \ldots, z_{n}\right)=\frac{1}{z_{n}} F_{n-1}\left(z_{1}, \ldots, z_{n-1}\right)+\mathrm{O}\left(z_{n}\right)$ as $z_{n} \rightarrow 0$.

(iv) $\left[\theta\left(z_{1}+\cdots+z_{n}\right) F_{n}\left(z_{1}, \ldots, z_{n}\right)\right]^{+}=0$ for all $n \geq 0$.

Proof. That the $F_{n}$ satisfy (i) and (ii) is clear from the definitions, and so is (iii) if we remember that $Q_{1}=0$ on $\mathscr{P}$. Property (iv) is immediate from (27) since none of the terms with $I \subsetneq\{1, \ldots, n\}$ contain all $n$ variables. For the uniqueness, we proceed by induction. Suppose that $F_{n}^{*}$ is another solution and that $F_{n^{\prime}}^{*}=F_{n^{\prime}}$ for $n^{\prime}<n$. Then $F_{n}^{*}=F_{n}+G_{n}$ where $G_{n}\left(z_{1}, \ldots, z_{n}\right)$ is $\mathrm{O}\left(z_{1} \cdots z_{n}\right)$ by (ii) and (iii), so $0=\left[\theta\left(z_{1}+\cdots+z_{n}\right) G_{n}\left(z_{1}, \ldots, z_{n}\right)\right]^{+}=\theta\left(z_{1}+\cdots+z_{n}\right) G_{n}\left(z_{1}, \ldots, z_{n}\right)$.

Equivalence of Theorem 4 and Theorem 1. The truth of equation (4) for all $f \in \Lambda_{*}=\mathbb{Q}\left[Q_{2}, Q_{3}, \ldots\right]$ is equivalent to its truth for the generating function

$$
f=\sum_{\substack{k_{1}, \ldots, k_{n} \geq 0 \\ k_{1}, \ldots, k_{n} \neq 1}} Q_{k_{1}} \cdots Q_{k_{n}} z_{1}^{k_{1}-1} \cdots z_{n}^{k_{n}-1}=\prod_{i=1}^{n}\left(W\left(z_{i}\right)-Q_{1}\right)
$$

for all $n \geq 0$, where $z_{1}, \ldots, z_{n}$ are formal variables. For this $f$ we have

$$
\left\langle e^{t \boldsymbol{\partial}} f\right\rangle_{q}=\left\langle\prod_{i=1}^{n}\left(e^{t z_{i}} W\left(z_{i}\right)-t\right)\right\rangle_{q}=\sum_{I \subseteq\{1, \ldots, n\}}(-t)^{n-|I|} e^{t z_{I}} F_{|I|}\left(\left\{z_{i}\right\}_{i \in I}\right),
$$


where $z_{I}$ denotes $\sum_{i \in I} z_{i}$. This in turn implies that

$$
\langle h(\boldsymbol{\partial}) f\rangle_{q}=\sum_{I \subseteq\{1, \ldots, n\}}(-1)^{n-|I|} h^{(n-|I|)}\left(z_{I}\right) F_{|I|}\left(\left\{z_{i}\right\}_{i \in I}\right)
$$

for any power series $h(z)$. Taking $h=\theta$, we obtain the equivalence of (4) and (31).

7. The leading coefficient of the $q$-bracket. In this section and the next one we will prove Theorems 2 and 3 as stated in the Introduction. The second of these implies the first, but we nevertheless prefer to prove each theorem separately. We make the preliminary remark that the extension of the "double factorial" $n ! !:=n \times(n-2) \times \cdots 3 \times 1(n \geq 1$ odd) to $(-1) ! !=1$ and $(-3) ! !=-1$ as given in Theorem 2 is the natural definition, forced by the functional equation $n ! !=n(n-2) ! !$. These values only play a role in Theorem 2 in the cases $k=0$ and $k=1$, where we know the theorem anyway, since $\langle 1\rangle_{q}=1$ and $\left\langle Q_{2}\right\rangle_{q}=\frac{1}{24} P$, but the consistency of the definition of the double factorial is important for the proof, which uses an induction over the weight.

We first restate Theorem 2 in a more convenient form. Let $T: \widetilde{M}_{*} \rightarrow \mathbb{Q}$ ("top term") be the map defined by $\phi=T(\phi)\left(-E_{2} / 12\right)^{k}+$ (lower-degree terms in $\left.E_{2}\right)$ if $\phi$ is a quasimodular form of weight $2 k$. Equivalently, $T$ can be defined as the ring homomorphism from $\mathbb{Q}[P, Q, R]$ to $\mathbb{Q}$ sending $P=E_{2}$ to -12 and $Q$ and $R$ to 0 . Then Theorem 2 can be rewritten as the identity

$$
T\left(\langle f\rangle_{q}\right)=-(2 k-3) ! ! \mu(f) \quad\left(\forall k \geq 0, f \in R_{2 k}\right) .
$$

To prove it we will need two lemmas, which we now state. The first is a simple identity.

Lemma 1. For any integers $k$ and $r$ with $0 \leq r \leq k$ we have

$$
\frac{(-1)^{r-1}(2 k-2 r-3) ! !}{2^{r} r !}=\frac{2^{k+1} k !}{(2 r+1) !} \operatorname{Res}_{t=-1}\left(\frac{t^{2 r+2} d t}{\left(1-t^{2}\right)^{k+1}}\right) .
$$

Proof. This formula, which can be written as a binomial coefficient identity by setting $t=-1+\epsilon$ and expanding the $(1-\epsilon)^{2 r+2}$ and $(1-\epsilon / 2)^{-k-1}$ by the binomial theorem, can be proved in several ways. We indicate two of these briefly, leaving the easy details to the reader. The most direct way is to use gamma function identities, Euler's beta integral and Cauchy's residue theorem to write

$$
\begin{aligned}
\frac{(2 k-2 r-3) ! !}{2^{r} r !} \frac{(2 r+1) !}{2^{k+1} k !} & =\frac{\Gamma\left(k-r-\frac{1}{2}\right) \Gamma\left(r+\frac{3}{2}\right)}{2 \pi \Gamma(k+1)} \\
& =\frac{1}{2 \pi} \int_{-\infty}^{\infty} \frac{u^{2 r+2} d u}{\left(1+u^{2}\right)^{k+1}}=i \operatorname{Res}_{u=i}\left(\frac{u^{2 r+2} d u}{\left(1+u^{2}\right)^{k+1}}\right),
\end{aligned}
$$

as desired (set $u=-i t$ ). However, this works only if $0 \leq r \leq k-1$, since the integral diverges when $r=k$. The second approach is to note that, if we denote the residue occurring in (33) by $R(r, k)$, then we have the two Pascal-triangle-like identities $R(r, k)-R(r+1, k)=R(r, k-1)$ (obvious) and $(2 r+1) R(r-1, k-1)+2 k R(r, k)=0$ (integration by parts), and using these one can reduce the general case to the case $(k, r)=(0,0)$ by induction over $r$ and $k$.

Lemma 2. For any $f_{1} \in \Lambda_{2 k+1}$ the polynomial $\mu\left(e^{t \boldsymbol{\partial}} f_{1}\right) \in \mathbb{Q}[t]$ is divisible by $(t+1)^{k+1}$.

Proof. From the generating function

$$
\sum_{n=0}^{\infty} \mu\left(e^{t \partial} Q_{n}\right) z^{n-1}=\mu\left(e^{t \partial} W(z)\right)=\mu\left(e^{t z} W(z)\right)=e^{t z} \sum_{n=0}^{\infty} \frac{1-n}{n !} z^{n-1}=\frac{1-z}{z} e^{(t+1) z}
$$


we get the closed formula

$$
\mu\left(e^{t \partial} Q_{n}\right)=\frac{(t+1)^{n}-n(t+1)^{n-1}}{n !} \quad(n \geq 0) .
$$

This is divisible by $(t+1)^{n-1}$ for all $n \geq 1$ and hence by $(t+1)^{n / 2}$ for all $n \geq 2$. Since both $e^{t \boldsymbol{\partial}}$ and $\mu$ are ring homomorphisms, it follows that $\mu\left(e^{t \boldsymbol{\partial}} f\right)$ is divisible by $(1+t)^{h / 2}$ for all $f \in \Lambda_{h}$. The lemma is just the case $h=2 k+1$ of this assertion.

Proof of Theorem 2. As in our derivation of the Bloch-Okounkov theorem from Theorem 1, we use the fact that the ring $\mathscr{R}$ is the sum (not direct) of the three subspaces $\mathbb{Q} \cdot 1, Q_{1} \mathscr{R}$, and $\boldsymbol{\partial}\left(\Lambda_{*}\right)$, so that it suffices to prove equation (32) for $f$ belonging to any one of these three spaces. For the first two, it is obvious: if $f=1$ we use $\langle 1\rangle_{q}=1, \mu(1)=1$ and $(-3) ! !=-1$, and if $f$ is divisible by $Q_{1}$ then the expression on the left is zero because $f$ vanishes on all partitions and the expression on the right because $\mu\left(Q_{1}\right)=0$ and $\mu$ is a ring homomorphism. If $f=\boldsymbol{\partial}\left(f_{1}\right)$ with $f_{1} \in \Lambda_{2 k+1}$, then we have equation (26). Applying the ring homomorphism $T$ to it, and using that $T\left(H_{2 r}\right)=(-1 / 2)^{r} / r$ ! (which follows easily by recursion from (13), and even more easily from the remark at the end of $\S 2$, which shows directly that $\left.T\left(\sum H_{n} z^{n}\right)=e^{-z^{2} / 2}\right)$, we find

$$
0=T\left(\sum_{r=0}^{\infty} H_{2 r}\left\langle\boldsymbol{\partial}^{2 r}(f)\right\rangle_{q}\right) \stackrel{?}{=} \sum_{r=0}^{\infty} \frac{(-1)^{r}(2 k-2 r-3) ! !}{2^{r} r !} \mu\left(\boldsymbol{\partial}^{2 r+1}\left(f_{1}\right)\right),
$$

where in the second line we have optimistically used (32) to rewrite each term $T\left(\langle\cdot\rangle_{q}\right)$ in terms of $\mu$. But now we see that it suffices to prove the vanishing of the expression on the right, since the equality indicated by "? =" is true by induction on the weight for all terms with $r>0$ and the $r=0$ term is precisely the identity we are trying to prove. And indeed, from Lemmas 1 and 2 we obtain

$$
\text { Right-hand side of }(34)=-2^{k+1} k ! \operatorname{Res}_{t=-1}\left(\frac{t \mu\left(e^{t \partial} f_{1}\right) d t}{\left(1-t^{2}\right)^{k+1}}\right)=0 \text {. }
$$

8. Relation of the $q$-bracket to the $\mathfrak{s l}_{2}$-action on quasimodular forms. We now turn to Theorem 3 , which we restate as

$$
\mathfrak{d}\langle f\rangle_{q}=\langle\mathcal{D} f\rangle_{q} \text { for all } f \in \mathscr{R}
$$

with $\mathcal{D}=\frac{1}{2}\left(\mathscr{D}-\partial^{2}\right)$, where $\boldsymbol{\partial}=\sum_{m=0}^{\infty} Q_{m} \partial / \partial Q_{m+1}$ as in Theorem 1 and $\mathscr{D}$ is the second order differential operator defined in (8). For the proof we need some properties of these operators.

Lemma 3. The differential operators $\mathscr{D}, \boldsymbol{\partial}, \mathcal{D}=\frac{1}{2}\left(\mathscr{D}-\partial^{2}\right)$ and $E=\sum_{m=1}^{\infty} m Q_{m} \partial / \partial Q_{m}$ (Euler operator on $\mathscr{R}$, acting on $\mathscr{R}_{k}$ as multiplication by $k$ ) satisfy the relations
(a) $\mathcal{D}\left(Q_{1} R\right) \subseteq Q_{1} R$.
(b) $\mathcal{D}$ and $\boldsymbol{\partial}$ commute.
(c) $\mathscr{D}\left(\Lambda_{*}\right) \subseteq \Lambda_{*}$.
(d) $\operatorname{Im}\left(\mathcal{D}+\frac{1}{2} E(E-3)\right) \subset \operatorname{Ker}(\mu) \quad(\mu: \mathscr{R} \rightarrow \mathbb{Q}$ as in Theorem 2$)$.
(e) $\left[\mathcal{D}, Q_{2}\right]=E-Q_{1} \partial-\frac{1}{2}$.

Proof. (a) From the definition of $\mathcal{D}$ we have

$$
2 \mathcal{D}=\sum_{k, \ell \geq 0}\left(\left(\begin{array}{c}
k+\ell \\
k
\end{array}\right) Q_{k+\ell}-Q_{k} Q_{\ell}\right) \frac{\partial^{2}}{\partial Q_{k+1} \partial Q_{\ell+1}}-\sum_{k \geq 0} Q_{k} \frac{\partial}{\partial Q_{k+2}}
$$


But the first sum can be replaced by one over $k, \ell \geq 1$, since the terms with $k \ell=0$ vanish. The resulting expression for $\mathcal{D}$ contains no derivatives with respect to $Q_{1}$ and hence is $\mathbb{Q}\left[Q_{1}\right]$-linear.

(b) It suffices to prove that $\mathscr{D}$ and $\boldsymbol{\partial}$ commute. We have

$$
\begin{aligned}
{\left[Q_{m} \frac{\partial}{\partial Q_{m+1}},\right.} & \left.Q_{k+\ell} \frac{\partial^{2}}{\partial Q_{k+1} \partial Q_{\ell+1}}\right]=\delta_{m, k+\ell-1} Q_{k+\ell-1} \frac{\partial^{2}}{\partial Q_{k+1} \partial Q_{\ell+1}} \\
& -\delta_{m, k+1} Q_{k+\ell} \frac{\partial^{2}}{\partial Q_{k+2} \partial Q_{\ell+1}}-\delta_{m, \ell+1} Q_{k+\ell} \frac{\partial^{2}}{\partial Q_{k+1} \partial Q_{\ell+2}}
\end{aligned}
$$

and hence, summing over all $k, \ell, m \geq 0$,

$$
[\boldsymbol{\partial}, \mathscr{D}]=\sum_{k, \ell \geq 0}\left(\left(\begin{array}{c}
k+\ell \\
k
\end{array}\right)-\left(\begin{array}{c}
k+\ell-1 \\
k
\end{array}\right)-\left(\begin{array}{c}
k+\ell-1 \\
\ell
\end{array}\right)\right) Q_{k+\ell-1} \frac{\partial^{2}}{\partial Q_{k+1} \partial Q_{\ell+1}}=0 .
$$

(c) This is obvious from the definition (8), since the terms with $k=0$ or $\ell=0$ vanish when applied to an element of $\Lambda_{*}$ and the factor $Q_{k+\ell}$ belongs to $\Lambda_{*}$ if $k$ and $\ell$ are both strictly positive.

(d) We have

$$
\begin{aligned}
2 \mathcal{D}+E(E-3)= & \sum_{k, \ell \geq 1}\left[\left(\begin{array}{c}
k+\ell-2 \\
k-1
\end{array}\right) Q_{k+\ell-2}-Q_{k-1} Q_{\ell-1}+k \ell Q_{k} Q_{\ell}\right] \frac{\partial^{2}}{\partial Q_{k} \partial Q_{\ell}} \\
& +\sum_{k \geq 1}\left[-Q_{k-2}+k(k-3) Q_{k}\right] \frac{\partial}{\partial Q_{k}} .
\end{aligned}
$$

The images under $\mu$ of the two expressions in square brackets are given respectively by:

$$
\frac{(3-k-\ell)-(2-k)(2-\ell)+(1-k)(1-\ell)}{(k-1) !(\ell-1) !}=0, \quad \frac{-(k-1)(3-k)+(k-3)(1-k)}{(k-1) !}=0,
$$

and since $\mu$ is a homomorphism, this shows that $\mu\left(\left(\mathcal{D}+\frac{1}{2} E(E-3)\right) f\right)=0$ for all $f \in \mathscr{R}$.

(e) Multiplying out (36) we find

$$
\mathcal{D}=\left(\frac{Q_{1}^{2}}{2}-Q_{2}\right) \frac{\partial^{2}}{\partial Q_{2}^{2}}+\left(E-Q_{1} \partial-\frac{1}{2}\right) \frac{\partial}{\partial Q_{2}}+\cdots
$$

where "..." denotes terms that contain no $Q_{2}$-derivatives and hence commute with multiplication by $Q_{2}$. The assertion follows from this and the relations $\left[E, Q_{2}\right]=2 Q_{2}$ and $\left[\boldsymbol{\partial}, Q_{2}\right]=Q_{1}$.

Proof of Theorem 3. We use for the third time the same principle as in the proofs of Theorem 2 and of the Bloch-Okounkov theorem, namely, that it is enough to prove (35) for the three cases $f=1$, $f \in Q_{1} R$, and $f \in \boldsymbol{\partial}\left(\Lambda_{*}\right)$. The first case is trivial, and the second follows from part (a) of Lemma 3, which implies that both sides of the equation vanish in this case, so we can assume that $f=\boldsymbol{\partial}\left(f_{1}\right)$ with $f_{1} \in \Lambda_{*}$. Then as before equation (26) holds, and if we apply $\mathfrak{d}$ to both sides of this equation, using that $\mathfrak{d}$ is a derivation and that it sends $H_{n}$ to $\frac{1}{2} H_{n-2}$ for all $n \geq 0$ (which is an easy consequence of the inductive definition (13) or of the final remark in $\S 2$ ), we find

$$
0=\mathfrak{d}\left(\sum_{r=0}^{\infty} H_{2 r}\left\langle\boldsymbol{\partial}^{2 r} f\right\rangle_{q}\right) \stackrel{?}{=} \sum_{r=0}^{\infty} H_{2 r}\left(\left\langle\mathcal{D}\left(\boldsymbol{\partial}^{2 r} f\right)\right\rangle_{q}+\frac{1}{2}\left\langle\boldsymbol{\partial}^{2 r+2} f\right\rangle_{q}\right)
$$


and, just as in the proof of Theorem 2, that by induction on the weight it suffices to prove the vanishing of the expression on the right in this formula. But by part (b) of Lemma 3 we have $2 \mathcal{D}\left(\partial^{2 r} f\right)+\partial^{2 r+2} f=$ $\mathscr{D}\left(\partial^{2 r+1} f_{1}\right)=\partial^{2 r+1}\left(\mathscr{D} f_{1}\right)$, and since $\mathscr{D} f_{1}$ belongs to $\Lambda_{*}$ by part (c) of the lemma, the required vanishing is an immediate consequence of Theorem 1 .

Note that in this proof we used only parts (a), (b) and (c) of Lemma 3. We use property (d) to prove the already-mentioned fact that Theorem 3 implies Theorem 2 . Indeed, from the definition of $T(\phi)$ ( = coefficient of $\left(-\frac{1}{12} E_{2}\right)^{k}$ in $\phi$ if $\left.\phi \in \widetilde{M}_{2 k}\right)$ it is clear that $T(\mathfrak{d} \phi)=-k T(\phi)$ for $\phi \in \widetilde{M}_{2 k}$, so from Theorem 3 and part (d) of the lemma we get

$$
-k T\left(\langle f\rangle_{q}\right)=T\left(\mathfrak{d}\langle f\rangle_{q}\right)=T\left(\langle\mathcal{D} f\rangle_{q}\right)=-(2 k-5) ! ! \mu(\mathcal{D} f)=k(2 k-3) ! ! \mu(f)
$$

for $f \in \mathscr{R}_{2 k}$ if Theorem 2 is true for $\mathcal{D} f$, showing that Theorem 2 then also holds for $f$ and hence is true by induction on the weight. Finally, property (e) is needed for the compatibility of Theorem 3 with the $\mathfrak{s l}_{2}$-action on $\widetilde{M}_{*}$ introduced in $\S 2$. Recall that this action was defined by the three derivations $D$ (differentiation with respect to $2 \pi i \tau$ ), $W$ (multiplication by the weight) and $\mathfrak{d}$ (differentiation with respect to $P=E_{2}$ ), with the commutation relations (12). The action of these derivations on $q$-brackets of elements from $\mathscr{R}$ is given by

$$
D\langle f\rangle_{q}=\left\langle Q_{2} f\right\rangle_{q}+\frac{P}{24}\langle f\rangle_{q}, \quad W\langle f\rangle_{q}=\langle E(f)\rangle_{q}, \quad \mathfrak{d}\langle f\rangle_{q}=\langle\mathcal{D}(f)\rangle_{q},
$$

where $E=\sum_{m=0}^{\infty} m Q_{m} \partial / \partial Q_{m}$ is the Euler operator on $\mathcal{R}$ as before. The first of these formulas (also, of course, pointed out and used in [3]) follows directly from the definition (2) of the $q$-bracket and the fact that $D(\eta)=P \eta / 24$, the second is the homogeneity statement in the Bloch-Okounkov theorem, and the third is equation (35). From these formulas and part (e) of Lemma 3 we get

$$
\begin{aligned}
(D \mathfrak{d}-\mathfrak{d} D+W)\langle f\rangle_{q} & =D\left(\langle\mathcal{D}(f)\rangle_{q}\right)-\mathfrak{d}\left(\left\langle Q_{2} f\right\rangle_{q}+\frac{P}{24}\langle f\rangle_{q}\right)+\langle E(f)\rangle_{q} \\
& =\left\langle Q_{2} \mathcal{D}(f)\right\rangle_{q}+\frac{P}{24}\langle\mathcal{D}(f)\rangle_{q}-\left\langle\mathcal{D}\left(Q_{2} f\right)\right\rangle_{q}-\frac{P}{24}\langle\mathcal{D}(f)\rangle_{q}+\left\langle\left(E-\frac{1}{2}\right) f\right\rangle_{q} \\
& =\left\langle Q_{1} \boldsymbol{\partial}(f)\right\rangle_{q}=0
\end{aligned}
$$

for any $f \in \mathscr{R}$, verifying the consistency of (37) with the $\mathfrak{s l}_{2}$-commutation relations (12).

We would like to extend this idea further by defining an action of $\mathfrak{s l}_{2}$ on shifted symmetric polynomials themselves, not just on their $q$-brackets. The first problem is that, as the above computation shows, the commutation relation would no longer hold with the obvious definitions, because the expression $Q_{1} \partial(f)$ vanishes only at the level of $q$-brackets, not in $\mathscr{R}$ itself. This can be fixed by noticing that (37) remains true if we replace the expressions $\left\langle Q_{2} f\right\rangle_{q}$ and $\langle\mathcal{D}(f)\rangle_{q}$ in the first and third formulas by $\left\langle\left(Q_{2}-\frac{1}{2} Q_{1}^{2}\right) f\right\rangle_{q}$ and $\left\langle\mathcal{D}(f)+Q_{1} \partial(\boldsymbol{\partial} f) / \partial Q_{2}\right\rangle_{q}$, respectively, since the $q$-bracket of any element of $Q_{1} \cdot \mathcal{R}$ is zero. Making this replacement and repeating the above computation, we indeed find that the unwanted term $Q_{1} \partial(f)$ goes away. This suggests mimicking the operators $D, W$ and $\mathfrak{d}$ by operators $\widehat{D}, \widehat{W}$ and $\widehat{\mathfrak{d}}$ that are defined initially for $f \in \mathscr{R}$ by

$$
\widehat{D}(f)=\left(Q_{2}-\frac{1}{2} Q_{1}^{2}+\frac{P}{24}\right) f, \quad \widehat{W}(f)=E(f), \quad \widehat{\mathfrak{d}}(f)=\mathcal{D}(f)+Q_{1} \frac{\partial}{\partial Q_{2}}(\boldsymbol{\partial} f)
$$

and then extended to $\widetilde{M}_{*} \otimes_{\mathbb{Q}} R=\mathbb{Q}\left[P, Q, R, Q_{1}, Q_{2}, \ldots\right]$ by

$$
\widehat{X}(\phi f)=X(\phi) f+\phi \widehat{X}(f) \quad \forall X \in \mathfrak{s l}_{2}, f \in \mathscr{R},
$$


where for simplicity we write simply $\phi f$ rather than $\phi \otimes f$ for the tensor product of $\phi \in \widetilde{M}_{*}$ and $f \in \mathscr{R}$. This indeed works, in the sense that these new operators satisfy the commutation relations (12) (with each $X$ replaced by $\widehat{X}$ ), so that they define an $\mathfrak{s l}_{2}$-action on the tensor product $\widetilde{M}_{*} \otimes \mathscr{R}$, but it is still not quite satisfactory because the subspace $\mathscr{R}$ of this tensor product is not preserved by the action, because of the term involving $P$ in (38). This term, of course, comes from the corresponding term in (38), i.e., the problem is due to the fact that the image of $\mathscr{R}$ is not closed under the action of $\mathfrak{s l}_{2}$ on quasimodular forms. But if we remember that the term $\frac{1}{24} P\langle f\rangle_{q}$ in (38) comes from differentiating the factor $\eta(\tau)^{-1}$ in (2), then the solution becomes clear: we must not work with the $q$-bracket defined in (1) (sum of $f$ over partitions normalized by the sum of 1 over partitions), but rather with the "naive $q$-bracket"

$$
\langle f\rangle_{q}^{\star}=\sum_{\lambda \in \mathscr{P}} f(\lambda) q^{Q_{2}(\lambda)}=\frac{\langle f\rangle_{q}}{\eta(\tau)} \in \frac{1}{\eta} \widetilde{M}_{*}
$$

(sum over partitions without any normalization). Then the $\tau$-derivative of the $q$-bracket is again a $q$-bracket. The price we have to pay is that this "naive $q$-bracket" no longer takes values in the ring $\widetilde{M}_{*}$, but in its isomorphic image $\eta^{-1} \widetilde{M}_{*}$, which is no longer a ring (but this is not very serious, since the $q$-bracket was not a ring homomorphism anyway), and also that we have to shift the grading in $\mathscr{R}$ from $k$ to $k-\frac{1}{2}$ in order for the new bracket map to be compatible with the grading. In other words, we can remove the term $\mathrm{Pf} / 24$ from the first equation in (38) at the expense of adding a term $-\frac{1}{2} f$ to the second equation, and this gives $\mathfrak{s l}_{2}$-actions on both $\mathcal{R}$ and its tensor product with $\widetilde{M}_{*}$ making the new $q$-bracket $\mathfrak{s l}_{2}$-equivariant. We summarize this discussion as:

Theorem 6. The operators $\widehat{W}, \widehat{D}$ and $\widehat{\mathfrak{d}}$ defined on $\mathscr{R}$ by

$$
\widehat{D}=Q_{2}-\frac{1}{2} Q_{1}^{2}, \quad \widehat{W}=E-\frac{1}{2}, \quad \widehat{\mathfrak{d}}=\mathcal{D}+Q_{1} \frac{\partial}{\partial Q_{2}} \circ \boldsymbol{\partial},
$$

and extended to $\widetilde{M}_{*} \otimes_{\mathbb{Q}} \mathscr{R}$ by (39), define actions of the Lie algebra $\mathfrak{s l}_{2}$ on these two spaces, and the modified q-bracket maps from $\mathscr{R}$ and $\widetilde{M}_{*} \otimes \mathscr{R}$ to $\eta^{-1} \widetilde{M}_{*}$ defined by $f \mapsto\langle f\rangle_{q}^{\star}$ and $\phi f \mapsto \phi\langle f\rangle_{q}^{\star}$ are both $\mathfrak{s l}_{2}$-equivariant.

9. Functions having quasimodular or nearly quasimodular $q$-brackets. The statement of the Bloch-Okounkov theorem, that the $q$-bracket of any shifted symmetric polynomial is quasimodular, seems like such a definitive statement that one feels this should be the end of the story. In this final section of my essay on their theorem, I would like to make clear that this is far from the case: not only is there a considerably larger class of functions having $q$-brackets that are only "nearly" quasimodular (explicitly, they are polynomials with quasimodular coefficients in the function $\log (q)_{\infty}$, which has a quasimodular derivative but is not quasimodular itself), but, what is perhaps even more surprising, there are very many more functions on partitions that have quasimodular $q$-brackets than just the elements of the ring $\Lambda_{*}$ or $\mathscr{R}$.

The simplest example of such a function is given by the moment function

$$
S_{k-1}(\lambda)=\sum_{i=1}^{j} \lambda_{i}^{k-1} \quad\left(k>0 \text { even, } \lambda=\left(\lambda_{1}, \ldots, \lambda_{j}\right) \in \mathscr{P}\right)
$$

$((k-1)$ st moment of the parts of $\lambda)$. Indeed, writing $\lambda$ as $1^{r_{1}} 2^{r_{2}} \cdots$, where $r_{m}=r_{m}(\lambda)$ denotes the number of parts of $\lambda$ equal to $m$, we have $S_{k-1}(\lambda)=\sum_{m \geq 1} r_{m}(\lambda) m^{k-1}$, so $\sum_{|\lambda|=n} S_{k-1}(\lambda)=$ 
$\sum_{m \geq 1} m^{k-1} N_{m}(n)$, where $N_{m}(n)=\sum_{|\lambda|=n} r_{m}(\lambda)$ denotes the total number of parts of size $m$ in all partitions of $n$. But the generating function of $N_{m}(n)$ for fixed $m \geq 1$ is clearly given by

$$
\sum_{n=0}^{\infty} N_{m}(n) q^{n}=\sum_{r_{1}, r_{2}, \cdots \geq 0} r_{m} q^{r_{1}+2 r_{2}+\cdots+m r_{m}+\cdots}=\frac{q^{m}}{\left(1-q^{m}\right)^{2}} \cdot \prod_{i \neq m} \frac{1}{1-q^{i}},
$$

a formula that can be written more succinctly in terms of $q$-brackets as $\left\langle N_{m}\right\rangle_{q}=q^{m} /\left(1-q^{m}\right)$. (This easy calculation can be found in many places in the literature.) Multiplying by $m^{k-1}$ and summing over all $m \geq 1$, we immediately obtain the formula

$$
\left\langle S_{k-1}\right\rangle_{q}=\sum_{m=1}^{\infty} m^{k-1}\left\langle r_{m}\right\rangle_{q}=\sum_{m=1}^{\infty} m^{k-1} \frac{q^{m}}{1-q^{m}}=G_{k}^{0} \quad(k \geq 2 \text { even }),
$$

where $G_{k}^{0}=G_{k}+B_{k} / 2 k$, the $k$ th Eisenstein series without its constant term. This equals $(1-P) / 24$ for $k=2$ and is the sum of a modular form of weight $k$ and a constant if $k>2$. Thus the $q$-bracket of $S_{k-1}$ is a non-homogeneous quasimodular form for every positive even integer $k$. But the functions $S_{k-1}$ themselves are not in general in the Bloch-Okounkov ring $\Lambda_{*}$ (or $\mathscr{R}$, which is the same as a space of functions on $\mathscr{P})$. For instance, one sees from the table given at the end of this paper that the unique element of $\Lambda_{4}$ having $G_{4}(=Q / 240)$ as its $q$-bracket is $Q_{2}^{2}+2 Q_{4}$. The $q$-bracket of the function $\lambda \mapsto S_{3}(\lambda)+\frac{1}{240}$ is also equal to $G_{4}$, but these two functions differ already on $\mathscr{P}_{2}$ : the function $Q_{2}^{2}+2 Q_{4}$ takes on the same value $\frac{1201}{240}$ on both partitions $(1,1)$ and $(2)$ of 2 , while the values of $S_{3}+\frac{1}{240}$ are $\frac{481}{240}$ and $\frac{1921}{240}$, which have the same sum $\frac{1201}{120}$ ( = the coefficient of $q^{2-\frac{1}{24}}$ in $G_{4} / \eta$ ) but are not the same.

We now describe a general construction producing a large collection of functions on partitions whose $q$-brackets are quasi-modular, but which in general are not in the ring $\mathscr{R}$. For each $n$, there are wellknown identifications of the set $\mathscr{P}_{n}$ of partitions of $n$ with both the set of conjugacy classes in the symmetric group $\mathfrak{S}_{n}$ and the set of irreducible representations of $\mathfrak{S}_{n}$. For $\lambda$ and $\mu$ in $\mathscr{P}_{n}$ we denote by $\chi_{\lambda}\left(C_{\mu}\right)$ the value of the character of the irreducible representation of $\mathfrak{S}_{n}$ associated to $\lambda$ at any element in the conjugacy class $C_{\mu}$ associated to $\mu$. We then define for any function $f: \mathscr{P} \rightarrow \mathbb{Q}$ a new function $m f: \mathscr{P} \rightarrow \mathbb{Q}$ by

$$
m f(\lambda)=\frac{1}{n !} \sum_{\mu \in \mathscr{P}_{n}}\left|C_{\mu}\right| \chi_{\lambda}\left(C_{\mu}\right)^{2} f(\mu) \quad\left(\lambda \in \mathscr{P}_{n}\right),
$$

where $\left|C_{\mu}\right|$ denotes the cardinality of the conjugacy class $C_{\mu}$. We call $m f$ the Möller transform of $f$.

Theorem 7. The map $m: \mathbb{Q}^{\mathscr{P}} \rightarrow \mathbb{Q}^{\mathscr{P}}$ preserves q-brackets.

Proof. The second orthogonality relation for characters says that $\frac{1}{n !} \sum_{\lambda \in \mathscr{P}_{n}} \chi_{\lambda}(C) \chi_{\lambda}\left(C^{\prime}\right)=\delta_{C, C^{\prime}}|C|^{-1}$ for any two conjugacy classes $C, C^{\prime}$ in $\mathfrak{S}_{n}$. Applying this to $C=C^{\prime}=C_{\mu}$, multiplying by $\left|C_{\mu}\right| f(\mu)$, and summing, we obtain $\sum_{\lambda \in \mathscr{P}_{n}} m f(\lambda)=\sum_{\mu \in \mathscr{P}_{n}} f(\mu)$ for every $f \in \mathbb{Q}^{\mathscr{P}}$, so $\langle m f\rangle_{q}=\langle f\rangle_{q}$. Note that we can also apply the first orthogonality relation $\frac{1}{n !} \sum_{\mu \in \mathscr{P}_{n}}\left|C_{\mu}\right| \chi_{\lambda_{1}}\left(C_{\mu}\right) \chi_{\lambda_{2}}\left(C_{\mu}\right)=\delta_{\lambda_{1} \lambda_{2}}$ with $\lambda_{1}=\lambda_{2}=\lambda$ to obtain that $m f=f$ whenever $f(\lambda)$ is a polynomial in $|\lambda|$, i.e., for all $f \in \mathbb{Q}\left[Q_{1}, Q_{2}\right] \subset \mathscr{R}$.

Theorem 7 implies that $\left\langle m^{j} f\right\rangle_{q}=\langle f\rangle_{q}$ for all $j \geq 0$. For $j<0$ the expression $m^{j} f$ does not make sense, since the map $m$ is not invertible. (The matrix $\left(\frac{\left|C_{\mu}\right|}{n !} \chi_{\lambda}(\mu)^{2}\right)_{\lambda, \mu \in \mathscr{P}_{n}}$ representing its action on $\mathbb{Q}^{\mathscr{P}_{n}}$ is singular already for $n=2$, where it equals $\frac{1}{2}\left(\begin{array}{ll}1 & 1 \\ 1 & 1\end{array}\right)$.) We can nevertheless define $m^{-r} \mathfrak{R}$ for $r>0$ as the space of functions $f: \mathscr{P} \rightarrow \mathbb{Q}$ for which $m^{r} f \in \mathscr{R}$. Then Theorem 7 immediately gives:

Corollary. Set $\mathcal{S}=\bigoplus_{j \in \mathbb{Z}} m^{j}(\mathscr{R}) \subseteq \mathbb{Q}^{\mathscr{P}}$. Then the q-bracket $\langle f\rangle_{q}$ is quasimodular for every $f \in \mathcal{S}$. 
We explain briefly the genesis of the transform $m$ and the reason for the name we have given it. The function $m f$ was discovered in the special case $f=S_{k-1}$ by Martin Möller in the course of his joint study with Dawei Chen of the Siegel-Veech constants for moduli spaces of flat surfaces. Möller and I then studied the function $T_{k-1}=m S_{k-1}$ numerically and found explicit formulas for it that prove in particular that $T_{k-1}$ belongs to the Bloch-Okounkov ring for every positive even integer $k$. This gives a different proof of the quasimodularity of the $q$-bracket of $T_{k-1}$ than the one coming from eq. (44) and Theorem 7. The functions $T_{k-1}$ have many further very interesting combinatorial and algebraic properties, with quite non-trivial proofs, which will be published separately in one or several papers by Chen, Möller and myself that are currently in preparation.

Actually, the function originally needed in the work of Chen and Möller was $m S_{-1}$. Here the formula that we found turned out to be related to an identity of Nekrasov and Okounkov (see [7] and [5]). In our language, this identity says that for any integer $m \geq 1$ the $q$-bracket of the function assigning to any partition the $m$ th elementary symmetric function of the reciprocal squares of the hook-lengths of the cells in its Young diagram is equal to $L^{m} / m$ !, where $L=-\log (q)_{\infty}$ is the nearly quasimodular form mentioned at the beginning of this section. More recently, I learned from Fernando Rodriguez Villegas of a (then still conjectural) formula he had discovered generalizing the Nekrasov-Okounkov formula, giving yet further examples of quasimodularity statements not included in the Bloch-Okounkov theorem. In the course of further discussions with him I tried many possible common generalizations of all of these results, but found only one that worked experimentally (i.e., led to quasimodular forms). The resulting statement turned out, following a suggestion of Möller, to be easily deducible from a theorem that was already in the literature. To end this section, and the paper, I will now state and prove this simple result, including for the reader's convenience also a self-contained proof of the earlier theorem.

We denote by $a(s)$ and $b(s)$ ( $a$ and $b$ for "Arm" and "Bein") the arm- and leg-lengths of a cell $s$ in the Young diagram of a partition $\lambda$, as defined in $\S 3$. It is actually more convenient to work with the modified quantities $\underline{a}(s)=a(s)+\frac{1}{2}$ and $\underline{b}(s)=b(s)+\frac{1}{2}$. (These are also more natural. For instance, the set $C_{\lambda}$ introduced in $\S 3$ is the union of the numbers $\underline{a}(s)$ and $-\underline{b}(s)$ for the diagonal cells, and the hook-length of any cell $s$ is simply the sum of $\underline{a}(s)$ and $\underline{b}(s)$.) For any even polynomial $P(x, y)$ in two variables (i.e., one satisfying $P(-x,-y)=P(x, y)$ ), we define an invariant $\mathcal{A}_{P}$ on partitions by

$$
\mathcal{A}_{P}(\lambda)=\sum_{s \in Y_{\lambda}} P(\underline{a}(s), \underline{b}(s))
$$

(sum over the cells of the Young diagram of $\lambda$ ). Then we will prove:

Theorem 8. The q-bracket of $\mathcal{A}_{P}$ is quasimodular for every even polynomial $P$.

As already stated, this result will be easy to prove using an earlier known result, discussed in detail in a 2008 article by G.-H. Han [5] and called there the Stanley-Elder-Bessenrodt-Bacher-Manivel or SEBBM theorem because of its somewhat complicated history as described in a paragraph of "Historical Remarks." (In a few words: the special case $b=0$ was discovered by Stanley in 1972, was rediscovered by Kirdar and Skyrme in 1982, by Elder in 1984, and by Hoare in 1986, and is known in the literature as Elder's theorem, while the general case was found by Bessenrodt [2] in 1998 and by Bacher and Manivel [1] in 2002 and rediscovered by Han in the paper in question.) Because both the formulation of the theorem and its proof are so spread out over the literature, we give a complete statement and self-contained proof here. For integers $a, b, n \geq 0$ denote by $N_{a, b}(n)$ the total number of cells with arm-length $a$ and leg-length $b$ in all Young diagrams of size $n$. Then the SEBBM result says:

Theorem (SEBBM). The number $N_{a, b}(n)$ depends only on $a+b$ and equals $N_{a+b+1}(n)$.

Example: The diagram below illustrates the equality $N_{1,1}(6)=N_{3}(6)$, with the Young diagrams on the left being those of size 6 with a marked (black) cell having (gray) arms and legs of length 1, and 
those on the right corresponding to the partitions $\underline{3}+3,3+\underline{3}, \underline{3}+2+1, \underline{3}+1+1+1$ of 6 having an (underlined, gray) part equal to 3 .
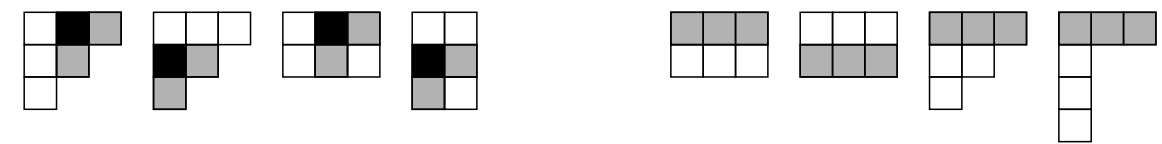

Proof. We already gave the generating function of $N_{m}$ in (43), so we only have to show that we get the same answer if we replace $N_{m}$ by $N_{a, b}$ for any $a, b \geq 0$ with $a+b=m-1$. Write $N_{a, b}(n)=\sum_{\lambda \vdash n} N_{a, b}(\lambda)$ with an obvious notation. Clearly each $N_{a, b}(\lambda)$ equals $\sum_{i, j>1} N_{a, b, i, j}(\lambda)$, where $N_{a, b, i, j}(\lambda)$ is defined as 1 if the cell $(i, j)$ belongs to the Young diagram $Y_{\lambda}$ and has arm- and leg-lengths $a$ and $b$, and as 0 otherwise. From the diagram below, in which any Young diagram $Y_{\lambda}$ having a cell with the parameters

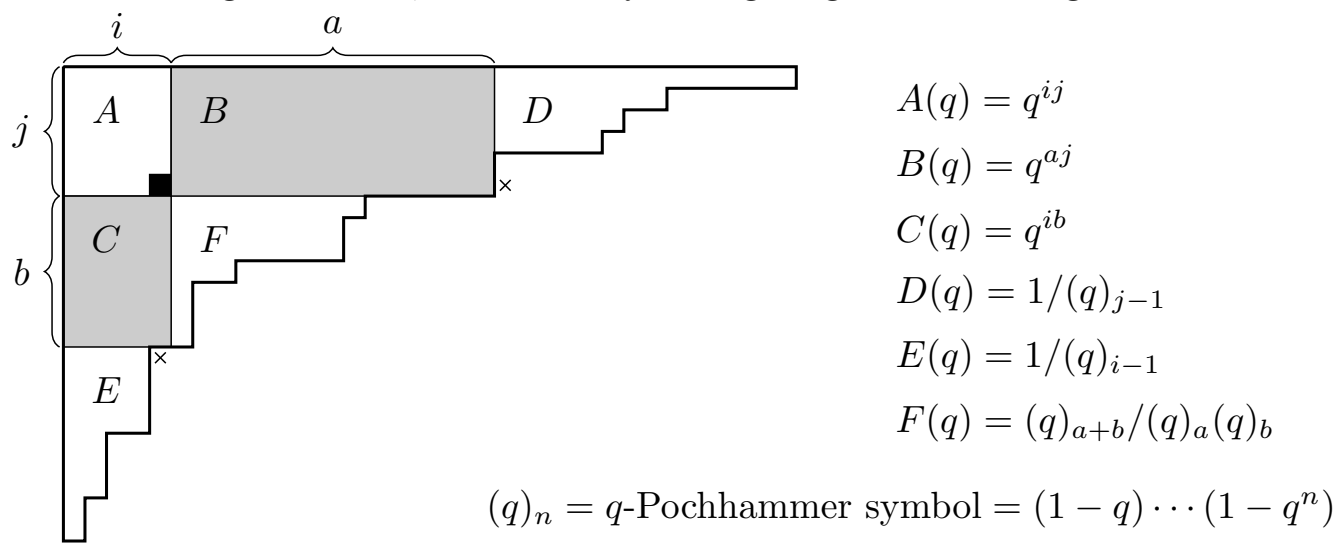

stated is partitioned canonically into three rectangular and three triangular regions $A, \ldots, F$, so that the total number $|\lambda|$ of cells in $Y_{\lambda}$ is decomposed correspondingly into six pieces, we see that

$$
\sum_{\lambda \in \mathscr{P}} N_{a, b, i, j}(\lambda) q^{|\lambda|}=A(q) B(q) C(q) D(q) E(q) F(q)
$$

with the values of the power series $A(q), \ldots, F(q)$ (in which the indices $a, b, i, j$ have been omitted from the notation) being as given in the diagram: the formulas for the first three (partitions into exactly $j$ or $b$ parts of size $i$ or $a$ ) are obvious, those for the next two (partitions into $<j$ parts and partitions into parts of size $<i$ ) almost equally so, and the one for $F$ (partitions into $\leq b$ parts of size $\leq a$ ) is also well-known and easy to derive. Now summing over $i$ and $j$ gives

$$
\begin{aligned}
\sum_{n=0}^{\infty} N_{a, b}(n) q^{n} & =\sum_{\lambda \in \mathscr{P}} N_{a, b}(\lambda) q^{|\lambda|}=\frac{(q)_{a+b}}{(q)_{a}(q)_{b}} \sum_{i, j \geq 1} \frac{q^{i j+a j+i b}}{(q)_{i-1}(q)_{j-1}} \\
& =\frac{(q)_{a+b}}{(q)_{b}(q)_{\infty}} \sum_{i \geq 1} \frac{(q)_{i+a-1}}{(q)_{a}(q)_{i-1}} q^{i b+i+a}=\frac{q^{a+b+1}}{1-q^{a+b+1}} \frac{1}{(q)_{\infty}}
\end{aligned}
$$

as desired, where for the equalities in the second line we have used the $q$-binomial theorem

$$
\sum_{n=0}^{\infty} \frac{(q)_{n+k}}{(q)_{n}(q)_{k}} x^{n}=\frac{1}{(1-x)(1-q x) \cdots\left(1-q^{k} x\right)} \quad(\text { in } \mathbb{Q}[[q, x]] \text { or for }|q|,|x|<1)
$$

twice, first with $(n, k, x)=\left(j-1, \infty, q^{i+a}\right)$ and then with $(n, k, x)=\left(i-1, a, q^{b+1}\right)$. 
Proof of Theorem 8. We first observe that if $P$ is an even polynomial, then the function

$$
P^{*}(m)=\sum_{\substack{a, b \geq 0 \\ a+b=m-1}} P\left(a+\frac{1}{2}, b+\frac{1}{2}\right) \quad(m \in \mathbb{N})
$$

is an odd polynomial of $m$. (This follows, for instance, from the fact that the power series

$$
\sum_{a+b=m-1}\left(e^{\left(a+\frac{1}{2}\right) x+\left(b+\frac{1}{2}\right) y}+e^{-\left(a+\frac{1}{2}\right) x-\left(b+\frac{1}{2}\right) y}\right)=\frac{\sinh m x-\sinh m y}{\sinh \frac{1}{2}(x-y)}
$$

is an odd function of $m$.) The SEBBM theorem and equation (43) then imply that

$$
\left\langle\mathcal{A}_{P}\right\rangle_{q}=(q)_{\infty} \sum_{a, b \geq 0} P\left(a+\frac{1}{2}, b+\frac{1}{2}\right) \sum_{n=0}^{\infty} N_{a, b}(n) q^{n}=\sum_{m=1}^{\infty} P^{*}(m) \frac{q^{m}}{1-q^{m}},
$$

which is a linear combination of functions $G_{k}^{0}$ with $k>0$ even and in particular quasimodular.

Theorem 8 gives us a large new collection of functions of partitions having quasimodular $q$-brackets. Numerical calculations show that these functions are in general neither in the Bloch-Okounkov ring nor in either its image or its inverse image under the Möller transform. Moreover, products of two or more of the functions $\mathcal{A}_{P}$ practically never have quasimodular $q$-brackets. These remarks together with Theorem 7 and its corollary give substance to the statement made at the end of the introduction that the space of functions on partitions whose $q$-brackets are quasimodular is much larger than the ring $\mathscr{R}$, and that it does not itself form a ring.

Appendix. Table of $q$-brackets up to weight 8. We give a list of $\langle f\rangle_{q}$ for all elements $f$ of $\Lambda_{*}$ of even weight $\leq 8$, using Ramanujan's notations $P=E_{2}, Q=E_{4}, R=E_{6}$.

$$
\begin{aligned}
& \langle 1\rangle_{q}=1 \\
& \left\langle Q_{2}\right\rangle_{q}=\frac{-P}{24} \\
& \left\langle Q_{2}^{2}\right\rangle_{q}=\frac{-P^{2}+2 Q}{576} \\
& \left\langle Q_{4}\right\rangle_{q}=\frac{5 P^{2}+2 Q}{5760} \\
& \left\langle Q_{2}^{3}\right\rangle_{q}=\frac{-3 P^{3}+18 Q P-16 R}{13824} \\
& \left\langle Q_{2} Q_{4}\right\rangle_{q}=\frac{15 P^{3}-6 Q P-16 R}{138240} \\
& \left\langle Q_{3}^{2}\right\rangle_{q}=\frac{5 P^{3}-3 Q P-2 R}{25920} \\
& \left\langle Q_{6}\right\rangle_{q}=\frac{-35 P^{3}-42 Q P-16 R}{2903040}
\end{aligned}
$$

$$
\begin{aligned}
& \left\langle Q_{2}^{4}\right\rangle_{q}=\frac{-15 P^{4}+180 Q P^{2}-320 R P+156 Q^{2}}{331776} \\
& \left\langle Q_{2}^{2} Q_{4}\right\rangle_{q}=\frac{75 P^{4}-144 Q P^{2}-128 R P+204 Q^{2}}{3317760} \\
& \left\langle Q_{2} Q_{3}^{2}\right\rangle_{q}=\frac{25 P^{4}-57 Q P^{2}+2 R P+30 Q^{2}}{622080} \\
& \left\langle Q_{2} Q_{6}\right\rangle_{q}=\frac{-175 P^{4}-168 Q P^{2}+160 R P+276 Q^{2}}{69672960} \\
& \left\langle Q_{3} Q_{5}\right\rangle_{q}=\frac{-35 P^{4}-21 Q P^{2}+26 R P+30 Q^{2}}{4354560} \\
& \left\langle Q_{4}^{2}\right\rangle_{q}=\frac{-2625 P^{4}-1260 Q P^{2}+1600 R P+2628 Q^{2}}{232243200} \\
& \left\langle Q_{8}\right\rangle_{q}=\frac{175 P^{4}+420 Q P^{2}+320 R P+228 Q^{2}}{1393459200}
\end{aligned}
$$




\section{Bibliography}

[1] R. Bacher and L. Manivel, Hooks and powers of parts in partitions. Sém. Lothar. Combin. 47, 2001/02, Article B47d, 11 pages.

[2] C. Bessenrodt, On hooks of Young diagrams. Ann. of Comb. 2 (1998), 103-110.

[3] S. Bloch and A. Okounkov, The character of the infinite wedge representation. Adv. Math. 149 (2000), 1-60.

[4] R. Dijkgraaf, Mirror symmetry and elliptic curves. In The Moduli Spaces of Curves (R. Dijkgraaf, C. Faber, G. van der Geer, eds.), Prog. in Math. 129, Birkhäuser, Boston (1995), 149-163.

[5] G.-N. Han, An explicit expansion formula for the powers of the Euler Product in terms of partition hook lengths [arXiv:math.CO/0804.1849v3] and The Nekrasov-Okounkov hook length formula: refinement, elementary proof, extension and applications [arXiv:math.CO/0805.1398].

[6] M. Kaneko and D. Zagier, A generalized Jacobi theta function and quasimodular forms. In The Moduli Spaces of Curves (R. Dijkgraaf, C. Faber, G. van der Geer, eds.), Prog. in Math. 129, Birkhäuser, Boston (1995), 165-172.

[7] N. Nekrasov and A. Okounkov, Seiberg-Witten theory and random partitions. In The unity of mathematics, Progr. Math. 244, Birkhäuser, Boston (2006), 525-596.

[8] R. Rudd, The string partition function for QCD on the torus, arXiv:hep-th/9407176.

[9] D. Zagier, Elliptic modular forms and their applications. In The 1-2-3 of Modular Forms: Lectures at a Summer School in Nordfjordeid, Norway (ed.: K. Ranestad), J. H. Bruinier, G. Harder, G. van der Geer and D. Zagier, Universitext, Springer-Verlag, Berlin-Heidelberg-New York (2008), 1-103. 